\title{
Nationale Förderprogramme und -strukturen in der Schweiz
}

\begin{abstract}
Seit dem Jahr 2000 können Schweizer Hochschulbibliotheken von nationalen Fördermassnahmen, die im Rahmen der Schweizerischen Universitätskonferenz bzw. Hochschulkonferenz zur Verfügung gestellt werden, profitieren. In bisher fünf Förderperioden wurden zahlreiche nationale Kooperations- und Innovationsprojekte erfolgreich initiiert und umgesetzt. Die ersten Projekte betrafen den Auf- und Ausbau des Konsortiums der Schweizer Hochschulbibliotheken (2000-2003 und 2004-2007). In der nächsten Förderperiode (2008-2012) und im Rahmen von e-lib.ch stand die zentrale Bereitstellung eines nationalen Zugangs $\mathrm{zu}$ wissenschaftlichen Inhalten unterschiedlicher Art im Vordergrund. Seit 2013 werden unter der Überschrift „Wissenschaftliche Information: Zugang, Verarbeitung und Speicherung“ Projekte von Bibliotheken und Anbietern von IT-Services im Hochschulbereich gemeinsam behandelt. Der Aufsatz umschreibt die Fördermassnahmen, bietet Einblick in die nationalen Rechtsgrundlagen und erläutert die Struktur der Organe und anderer Akteure im Förderumfeld.
\end{abstract}

\section{Einführung}

Dieser Artikel verfolgt zwei Ziele: Einerseits bietet er einen Überblick über die wichtigsten nationalen Fördermassnahmen, von denen Schweizer Hochschulbibliotheken profitieren. Andererseits präsentiert er die relevanten nationalen Rechtsgrundlagen sowie die Organisationsstruktur im tertiären Bildungssektor. Viele der Projekte, die im Rahmen dieser Fördermassnahmen lanciert worden sind, bilden den Inhalt einzelner Autorenbeiträge im vorliegenden Sammelband. Sie werden entsprechend hier nur summarisch erwähnt oder am Ende des Beitrags aufgelistet (Tab. 2). Für diesen Beitrag relevant ist vor allem die Zuordnung zu den einzelnen Förderperioden, das Verständnis von Governancestrukturen und die Finanzierung (vgl. Abb.1). Von besonderer Bedeutung sind die zwei Förderprogramme SUK P-2 (2013-2016) und P-5 (2017-2020) mit dem gemeinsamen Titel „Wissenschaftliche Information: Zugang, Verarbeitung und Speicherung“ (vgl. hierzu auch die Ausführungen von Gabi Schneider in diesem Band).

Vorliegender Beitrag beschränkt sich auf die Präsentation nationaler Programme. Natürlich gibt es in der Schweiz auch wichtige Innovationsprojekte ausserhalb 
dieses Rahmens ${ }^{1}$; sie stehen hier allerdings nicht im Mittelpunkt. Für nationale Vorhaben stehen seit dem Jahr 2000 Fördermittel im Rahmen projektgebundener Beiträge des Bundes zur Verfügung. Frühere nationale Projekte wurden über Sonderkredite oder den Berufsverband gefördert und koordiniert.

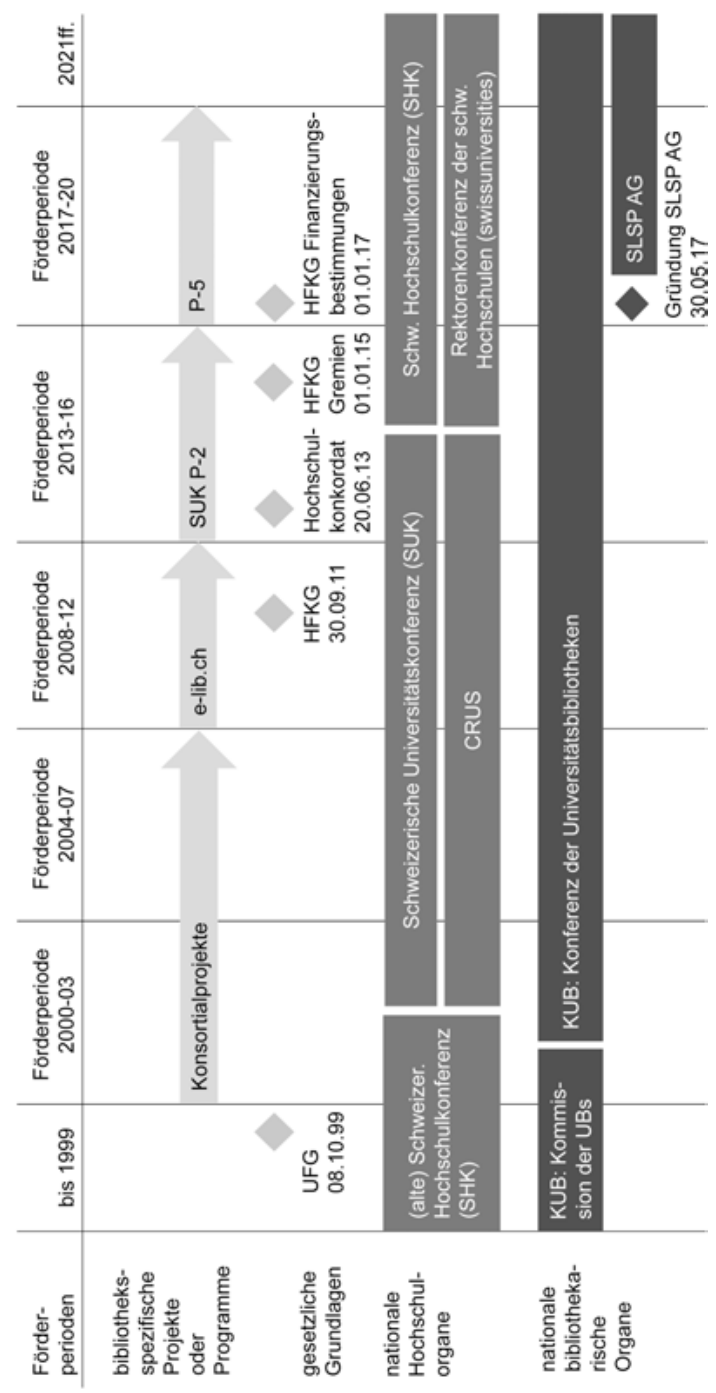

Abb. 1: Zeitleiste zu den nationalen Förderprogrammen, Rechtsgrundlagen und Organen

$1 \mathrm{Zu}$ erwähnen sind hier beispielsweise der Aufbau von institutionellen Repositorien, die Förderung von E-Learning-Angeboten oder alternativen Publikationsplattformen. 


\section{Nationale Förderprogramme}

\section{Situation bis 1999}

Mit der im Jahr 1897 gegründeten Vereinigung Schweizerischer Bibliothekare (VSB) verfügt die Schweiz über die älteste bibliothekarische Berufsvereinigung mit Kontinuität auf dem europäischen Kontinent. ${ }^{2}$ Insofern könnte man erwarten, dass die Schweizer Bibliotheken auf eine lange und intensive Kooperation zurückblicken können. Aber in der Realität wechseln sich „bibliothekarische Leistungen und Versäumnisse“ ab, wie Robert Barth sorgfältig recherchiert und zusammenfasst. $^{3}$

Als ältestes Kooperationsprojekt der Schweizer Bibliotheken gilt allgemein das Zeitschriften-Verzeichnis der schweizerischen Bibliotheken, umfassend die im Jahr 1902 gehaltenen Periodica und Serien (VZ 1) aus dem Jahr 1904. ${ }^{4}$ Die Schaffung eines allgemeinen Gesamtkataloges dauerte bedeutend länger, konnte dann aber aufgrund eines Kredites der Eidgenössischen Räte an die Landesbibliothek im Jahr 1928 an die Hand genommen werden. ${ }^{5}$ Der Aufbau von landesweiten Nachweisinstrumenten setzte ein einheitliches und gemeinsames Regelwerk für die Katalogisierung voraus, was gerade in einem mehrsprachigen Land eine besondere Herausforderung darstellte. Entsprechend wurde die Veröffentlichung der Katalogisierungsregeln als eine der „bleibenden Leistungen der VSB in den 1970er Jahre“ gewürdigt. ${ }^{6}$ Der Erfolg dieses gemeinsamen Regelwerks war aber leider nicht bleibend und fiel sozusagen der Automatisierung ab den 80er Jahren zum Opfer. Das heisst, die Verbünde wählten für ihre Arbeit divergierende Formate, die ihnen bessere Möglichkeiten des Datenaustausches mit dem jeweiligen gleichsprachigen Ausland gewährten.

Insgesamt führten die Automatisierung und das Aufkommen der Verbünde zu einer Zersplitterung des Schweizer Bibliothekswesens in regionale oder überregionale Netzwerke, die mitunter auch in Konkurrenz zueinander standen. Aufgrund unterschiedlicher Bibliothekssysteme, Kooperationsmodelle sowie Regelwerke kam es insbesondere zwischen der West- und Deutschschweiz zu einem

2 Robert Barth, unter Mitw. von Gabi Schneider: Bibliotheken, Bibliothekarinnen und Bibliothekare in der Schweiz. Hundert Jahre bibliothekarischer Branchenverband 1897-1997. Cent ans d'association professionnelle 1897-1997. Bibliothèques et bibliothécaires en Suisse. Vevey 1997, S. 25; vgl. hierzu auch den Beitrag „Bibliotheksverband“ von Herbert Staub in diesem Band.

3 Ebd. S. 57-77.

4 Ebd. S. 57.

5 Ebd.S. 58.

6 Fredy Gröbli zitiert aus Barth/Schneider, Bibliotheken (wie Anm. 2), S. 61. 
Auseinanderdriften der Hochschulbibliotheken, wobei die Zersplitterung nicht immer genau der Sprachgrenze folgte. In diesem komplexen Gefüge gelang es auch der Nationalbibliothek nicht, eine gemeinsame Richtung in der Erschliessungsfrage vorzugeben und durchzusetzen.

Dieses Auseinanderdriften der Hochschulbibliotheken in den 80er und 90er Jahren hängt auch damit zusammen, dass der Bund zwar regelmässig Kommissionen für die Bearbeitung von bibliothekarischen oder informationswissenschaftlichen Fragen einsetzte, aber keine finanzielle Unterstützung anbieten konnte oder wollte. Sogar konkrete Projekte, die der Bund selbst als wesentlich bezeichnete - wie zum Beispiel der Aufbau von Sammelschwerpunkten oder eines Instituts für Informationswissenschaften - konnten nicht umgesetzt werden. Besonders schmerzlich für die Bibliotheken war die Ablehnung des Kulturförderungsartikels durch das Stimmvolk im Jahr 1994. Demzufolge fanden die Bibliotheken keine Verankerung in der Hochschulförderung des Bundes. ${ }^{7}$ Hochschulbibliotheken blieben letztlich von ihren Trägern - Bund oder Kanton - abhängig und hatten bis zur Jahrtausendwende keinen Zugang zu gemeinsamen Trägerstrukturen oder Förderprogrammen. Entsprechend schwierig war auch die Initiierung und Durchführung gemeinsamer Vorhaben und Projekte.

\section{Projektgebundene Beiträge 2000-2003 und 2004-2007: Konsortialprojekte}

Erstmals wurden im Jahre 2000, gestützt auf dem Universitätsförderungsgesetz (UFG) vom 08. Oktober 1999, projektgebundene Beiträge als neues Instrument zur Förderung der Hochschulentwicklung in der Schweiz eingeführt. ${ }^{8}$ Mit diesen Beiträgen wurden universitäre Kooperationsprojekte und Innovationen von gesamtschweizerischer Bedeutung unterstützt. Die Schweizerische Universitätskonferenz (SUK) war von Anfang an für die Ausschreibung und die Vergabe dieser Beiträge zuständig. Für Bibliotheken lag Anfang der 2000er Jahre der Fokus auf der Gründung und dem Ausbau des nationalen Konsortiums der Schweizer Hochschulbibliotheken. Grundlage hierfür bot eine von der damaligen Kommission der Universitätsbibliotheken (KUB) in Auftrag gegebene Projektstudie, welche die

7 Vgl. Barth/Schneider, Bibliothekenn (wie Anm. 2), S. 135-138.

8 Dora Fitzli u.a.: Schlussevaluation der mit projektgebundenen Beiträgen nach UFG geförderten Projekte 2008-2011. Schlussbericht. Bern 2012 (https://www.shk.ch/wp-content/uploads/2017/ 01/PgB-2008-2012_Evaluation-econcept.pdf). Alle Links in diesem Beitrag wurden am 26.03.2018 überprüft. 
Schaffung eines gesamtschweizerischen Konsortiums der Hochschulbibliotheken dringend vorschlug. ${ }^{9}$

Die Versammlung der Schweizerischen Hochschulkonferenz (SHK) hatte diesen Vorschlag der KUB zustimmend zur Kenntnis genommen und einen Grundsatzbeschluss über die Projektrealisierung und -finanzierung gefällt. Anfang 2000 folgte die Ausschüttung der ersten projektgebundenen Mittel für das Konsortium. ${ }^{10}$

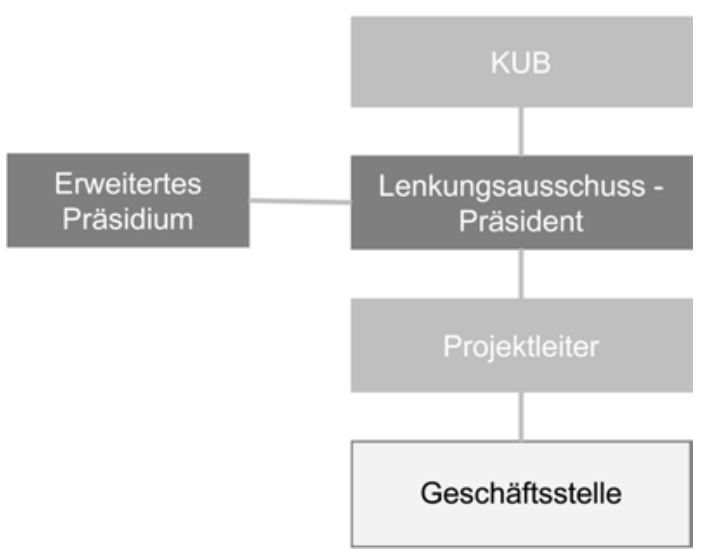

Abb. 2: Organstruktur des Konsortiums der Schweizerischen Hochschulbibliotheken (Stand 2011) ${ }^{11}$

Im Rahmen des Konsortiums wurden zwei Projekte oder Module umgesetzt: das Modul Lizenzen (2000-2005) und das Modul E-Archiving (2002-2005). Diese vom nationalen Konsortium lancierten Projekte gelten gemeinhin als die ersten mit Bundesmitteln geförderten Bibliotheksprojekte der Schweiz. ${ }^{12}$ Sie erstrecken sich über die ersten zwei Phasen der projektgebundenen Beiträge des Bundes, 20002003 und 2004-2007. Da zu diesem Zeitpunkt kantonale Universitäten, ETH und Fachhochschulen noch kein gemeinsames Förderdach besassen, erfolgte die Finanzierung über getrennte Kanäle.

9 Alice Keller: Konsortienbildung in der Schweiz. In: Zeitschrift für Bibliothekswesen und Bibliographie, Sonderheft 77 (1999), S. 147-160.

10 Vgl. hierzu auch den Beitrag „Konsortium“ von Rafael Ball und Pascalia Boutsiouci in diesem Band.

11 Pascalia Boutsiouci: Rechtliche Aspekte des Konsortiums der Schweizer Hochschulbibliotheken. In: Anne Cherbuin u. a. (Hrsg.): Digitale Bibliotheken und Recht. Tagung der Vereinigung der juristischen Bibliotheken der Schweiz. Zürich 2011, S. 79-88, hier Abbildung 1.

12 Wolfram Neubauer, Arlette Piguet: The Long Road to Becoming a "Consortium of Swiss University Libraries“. In: LIBER Quarterly 11/1 (2001), S. 87-97. 
Für die strategische Ausrichtung des Konsortiums wurde ein Lenkungsausschuss eingesetzt, der an die KUB berichtete (Abb. 2). Für die Umsetzung der Projektvorhaben wurde eine Geschäftsstelle an der ETH Zürich aufgebaut. Seit 2006 erfolgt die Finanzierung der Lizenzen und der Geschäftsstelle ausschliesslich aus Beiträgen der beteiligten Partner bzw. Bibliotheken.

\section{e-lib.ch: Elektronische Bibliothek Schweiz (2008-2012)}

Im Jahre 2008 setzte eine neue Förderperiode ein, die den Zeitraum 2008 bis 2011, mit Verlängerung bis 2012, abdeckte. Massgeblich für die Bibliotheken war hier das nationale Innovations- und Kooperationsprojekt e-lib.ch: Elektronische Bibliothek Schweiz, das als „logische Weiterentwicklung und aufbauend auf den im Konsortium gemachten Erfahrungen“ bezeichnet wurde und darüber hinaus eine Ausweitung der Themen und der Partner bedeutete. ${ }^{13}$

Ausgangspunkt für das Projekt e-lib.ch war ein Projektantrag der Bibliotheken an die Schweizerische Universitätskonferenz (SUK). In diesem Rahmen wurden anfänglich CHF 7 Mio. für bibliotheksspezifische Innovations- und Kooperationsprojekte zur Verfügung gestellt. Die Beiträge für die Teilnahme von ETH und Fachhochschulen wurden vom ETH-Rat bzw. Staatssekretariat für Bildung, Forschung und Innovation gesondert gesprochen (siehe Tab. 1). ${ }^{14}$ Ursprünglich war das Projekt für die Laufzeit 2008-2011 geplant; es wurde dann aber um zwölf Monate bis Ende 2012 verlängert und die Beiträge wurden entsprechend erhöht.

13 Susanne Benitz, Nadja Böller: e-lib.ch. Elektronische Bibliothek Schweiz - eine Erfolgsgeschichte? In: b.i.t.online 16/2 (2013), S. 97-102, hier S. 97.

14 E-lib.ch wurde für die kantonalen Universitäten durch die Schweizerische Universitätskonferenz (SUK) finanziert. Die Teilnahme der Institutionen des ETH-Bereichs wurde vom ETH-Rat getragen. Die Fachhochschulen erhielten Unterstützung vom Staatssekretariat für Bildung, Forschung und Innovation (SFBI, ehemals BBT - Bundesamt für Berufsbildung und Technologie). 
Tab. 1: Finanzierung von e.lib.ch, differenziert nach Hochschultyp ${ }^{15}$

\begin{tabular}{llllr}
\hline & SUK & ETH-Rat & SFBI & Total \\
\hline Hochschultyp & $\begin{array}{l}\text { Kantonale } \\
\text { Universitäten }\end{array}$ & ETH-Bereich & Fachhochschulen & \\
\hline Laufzeit 2008-2011 & CHF 7 Mio. & CHF 2 Mio. & CHF 1 Mio. & CHF 10 Mio. \\
\hline Verlängerung 2012 & CHF 1,2 Mio. & CHF 0,4 Mio. & CHF 0,2 Mio. & CHF 1,8 Mio. \\
\hline & & & & CHF 11,8 Mio. \\
\hline
\end{tabular}

Übergreifendes Ziel von e-lib.ch war die zentrale Bereitstellung eines nationalen Zugangs zu wissenschaftlichen Inhalten unterschiedlicher Art. Zur Erreichung dieses Ziels wurde, wie oben erwähnt, ein Rahmenbudget zur Verfügung gestellt. Die Auswahl der einzelnen Projekte erfolgte durch den Lenkungsausschuss, dessen Selektionskriterien sich an den aktuellen Entwicklungen im globalen Informations- und Wissenschaftsbereich orientierten. ${ }^{16}$

Ähnlich komplex wie die Finanzierung gestaltete sich auch die Governancestruktur (Abb.3). Das Projekt wurde im Auftrag der Rektorenkonferenz der Schweizer Universitäten (CRUS) auf nationaler Ebene durch die Konferenz der Universitätsbibliotheken (KUB) durchgeführt. Diese setzte einen Lenkungsausschuss ein und übertrug die Projektleitung und Koordinationsstelle der ETHBibliothek (vgl. Abb. 2). Wie bereits bei den Konsortialprojekten der vorherigen Phasen kam auch hier der ETH-Bibliothek eine zentrale Rolle zu.

Inhaltlich hatte e-lib.ch zum Ziel, eine Reihe einzelner Applikationen und Dienstleistungen neu zu entwickeln und unter einem Portal zu bündeln. Des Weiteren ging es darum, bereits laufende oder in der Initiierungsphase befindliche Anwendungen in das Portal einzubinden. Und schliesslich sollte sich e-lib.ch mittelfristig als nationaler Einstiegspunkt für die Recherche in die elektronischen Informationsangebote etablieren.

„E-lib.ch“ stand einerseits als Name für das Gesamtprojekt bestehend aus 20 Teilprojekten in sechs Kategorien ${ }^{17}$ und andererseits als Benennung des übergreifenden Webportals, das die Bündelung aller Inhalte leistete. Die inhaltsrele-

15 Maximiliane Okonnek: e-lib.ch: Elektronische Bibliothek Schweiz. Konferenzbeitrag, Kongress BIS, Konstanz 2012, hier Folien 13-14 (http://dx.doi.org/10.3929/ethz-a-009766873).

16 Elektronische Bibliothek Schweiz. Bibliothèque électronique suisse. Entwicklung und Stand des Infrastrukturprojekts e-lib.ch. Fragen an Wolfram Neubauer. In: arbido 3/2011, S. 8-11.

17 Sechs Kategorien von e-lib.ch: Digitale Sammlungen, Werkzeuge, Fachportale, Wissenschaftliches Publizieren, Dokumentenserver, Informationskompetenz. 
vanten Arbeiten wurden von verschiedenen Partnern geleistet, während die ETHBibliothek das Webportal zur Verfügung stellte. Eine Auflistung der Projekte findet sich am Ende dieses Beitrags.

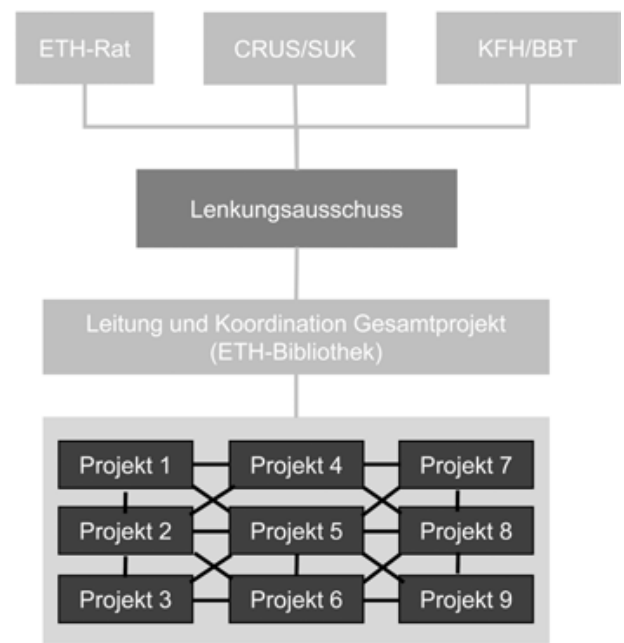

Abb. 3: Governancestruktur von e-lib.ch ${ }^{18}$

\section{SUK P-2 „Wissenschaftliche Information: Zugang, Verarbeitung und Speicherung“" (2013-2016)}

In der Beitragsperiode 2013 bis 2016 verfügten die Bibliotheken nicht mehr über ein ,eigenes Programm', sondern wurden zusammen mit Anbietern von IT-Services im Hochschulbereich - namentlich SWITCH - in einem gemeinsamen Rahmenprogramm behandelt. So umfasste das für Bibliotheken relevante Programm SUK P-2 „Wissenschaftliche Information: Zugang, Verarbeitung und Speicherung“ auch Handlungsfelder wie E-Publishing, Data Management, Identity Management, Cloud Computing und E-Learning. Unter dem Begriff ,organisatorische Aspekte، sollte ausserdem die Weiterführung bzw. Einbindung bestehender Applikationen und Services aus e-lib.ch und den Konsortialaktivitäten sichergestellt werden. ${ }^{19}$

18 Matthias Töwe: Elektronische Bibliothek Schweiz E-lib.ch. Zwischenbilanz und Ausblick. Konferenzbeitrag, KUB, Bern, 17.11.2010, hier Folie 6 (https://doi.org/10.3929/ethz-a-006246804). 19 Vgl. Programm SUK 2013-2016 P-2,,Wissenschaftliche Information: Zugang, Verarbeitung und Speicherung“. Bündelung der Kräfte in der wissenschaftlichen Information. Nationale Strategie. 
Mit einer Gesamtsumme von insgesamt CHF 45 Mio. in Form von projektgebundenen Beiträgen war das Programm im Vergleich zu früheren Rahmenkrediten grosszügig dotiert, sollte aber von Bibliotheken und IT-Providern gemeinsam ausgeschöpft werden. ${ }^{20}$ Die Beiträge stellten eine Anschubfinanzierung dar; im Regelfall wurde eine Eigenbeteiligung von 50\% vorausgesetzt. Wichtiges Auswahlkriterium bei der Evaluation der Anträge war der Entwurf eines zukunftsfähigen Geschäftsmodells. Die inhaltliche Ausrichtung des Programms wird im Beitrag von Gabi Schneider in diesem Band erläutert.

In der Organisationsform präsentierte sich das SUK P-2-Programm gegenüber der letzten Förderperiode um eine Stufe komplexer (Abb. 4). Die Projektleitung war organisatorisch nicht mehr einer Bibliothek angegliedert. Der von der Rektorenkonferenz CRUS eingesetzte Lenkungsausschuss verantwortete die Durchführung des Programms gegenüber der SUK und entschied über die Umsetzungsvorschläge zur Bewilligung bzw. Ablehnung von einzelnen Projektanträgen.

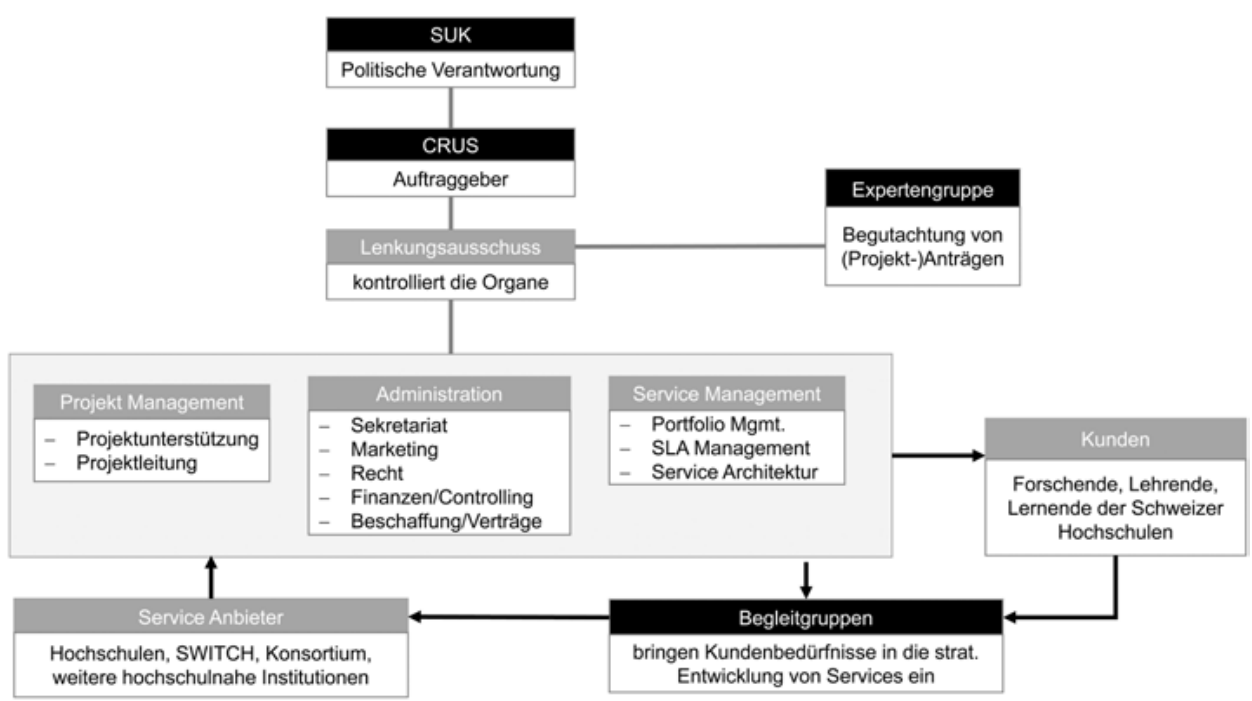

Abb. 4: Programmführung SUK P-2 21

Von der SUK bewilligt am 03.04.2014 (https://www.swissuniversities.ch/fileadmin/swissuniversi ties/Dokumente/Organisation/SUK-P/SUK_P-2/SUK_P-2_NationaleStrategie_20140403_DE.pdf). 20 Die gesamthaft CHF 45 Mio. setzten sich wie folgt zusammen: SUK CHF 36 Mio., ETH-Rat CHF 7 Mio., Fachhochschulen CHF 2 Mio.

21 Vgl. Programm SUK 2013-2016 P-2 (wie Anm. 19), S. 16. 


\section{P-5 „Wissenschaftliche Information: Zugang, Verarbeitung und Speicherung“(2017-2020)}

Obwohl sich Rechtsgrundlage und Organstruktur im Hochschulbereich seit Inkraftsetzung des neuen Hochschulförderungs- und -koordinationsgesetzes am 01. Januar 2015 grundlegend verändert haben, stellt die neue Förderphase 20172020 eine nahtlose Fortsetzung des letzten Programms dar. ${ }^{22}$ Portfolio und Projektorganisation der letzten Förderperiode werden in der eingeschlagenen Richtung weiterentwickelt und mit Blick auf angrenzende Vorhaben konsolidiert. Grundlage für die Umsetzung des Programms bildet seit November 2016 die Umsetzungsstrategie 2017 bis 2020. Hiermit werden die Umsetzungsmassnahmen aus dem White Paper von 2014 an den aktuellen Stand des Programms angepasst und stellen so die Grundlage für Projektanträge dar. ${ }^{23}$

Das neue Programm P-5 ist mit einem Bundesbeitrag von CHF 30 Mio. dotiert - also mit bedeutend weniger Mitteln als in der letzten Förderphase. Dabei ist zu berücksichtigen, dass SUK P-2 aufgrund der Auflage, vor der Vergabe von Projektbeiträgen eine Gesamtstrategie zu entwickeln, nicht alle Mittel ausschöpfen konnte. Diese Restmittel können nun dazu verwendet werden, bereits in der vorherigen Förderphase bewilligte Projekte mit einer Laufzeit über 2016 hinaus in P-5 zu decken.

In konsequenter Fortsetzung der vorherigen Förderperiode werden die Bibliotheksprojekte nicht gesondert behandelt. Das Programm P-5 soll die Leistungen von Bibliotheken, IT-Services und Scientific IT explizit zu einer Basis für Science 2.0 und Open Science verbinden. Darüber hinaus soll für den Anstoss des dauerhaften Betriebs, für die Verwaltung und für den Zugang zu Diensten eine hochschulübergreifende Governance (Projektbezeichnung „nationale Organisation“) mit einer Serviceplattform aufgebaut werden. ${ }^{24}$ Das Programm wird im Beitrag von Gabi Schneider umfassend vorgestellt.

22 Die rechtliche Basis für die Vergabe von projektgebundenen Beiträgen für Kooperations- und Innovationsprojekte basiert nun auf Art. 59 HFKG.

23 Vgl. Programm SUK 2013-2016 P-2 „Wissenschaftliche Information: Zugang, Verarbeitung und Speicherung “. White Paper for a Swiss Information Provisioning and Processing Infrastructure 2020. Veröffentlicht 2014 (https://www.swissuniversities.ch/fileadmin/swissuniversities/Do kumente/Organisation/SUK-P/SUK_P-2/WhitePaper_V1.1-DE.pdf).

24 Vgl. Projektgebundene Beiträge 2017-2020 nach HFKG: Projektantrag „Wissenschaftliche Information: Zugang, Verarbeitung und Speicherung“. Eingereicht an swissuniversities im Februar 2016 (https://www.swissuniversities.ch/fileadmin/swissuniversities/Dokumente/Hochschul politik/Pgb_17-20/PA_05_Wissenschaftliche_Information.pdf). 


\section{Nationale Rechtsgrundlagen und Organe des Hochschulsektors}

\section{Situation vor 2015: Schweizerische Universitätskonferenz (SUK) und Rektorenkonferenz CRUS}

Vorgängerin der heutigen Hochschulkonferenz war die Schweizerische Universitätskonferenz (SUK). Allerdings waren hierin nur die universitären Hochschulen vertreten - ohne Fachhochschulen und Pädagogische Hochschulen. Die SUK wurde, gestützt auf das Universitätsförderungsgesetz vom 08. Oktober 1999 (UFG), im Jahr 2001 durch die Vereinbarung zwischen Bund und Universitätskantonen über die Zusammenarbeit im universitären Hochschulbereich als Nachfolgeorganisation der (älteren) Schweizerischen Hochschulkonferenz gegründet. Die SUK arbeitete eng mit der CRUS, der ehemaligen universitären Rektorenkonferenz, zusammen. Die SUK verfügte über bindende Entscheidungskompetenzen in definierten Bereichen und war unter anderem zuständig für die Gewährung von projektgebundenen Beiträgen. Im Rahmen der meist vierjährigen Planungsphasen wurden die oben genannten Vorhaben Konsortialprojekte, e-lib.ch sowie SUK P-2 substantiell durch die SUK unterstützt. Mit der Inkraftsetzung des Hochschulförderungs- und -koordinationsgesetzes (HFKG) am 01. Januar 2015 wurde die SUK aufgelöst bzw. in die Schweizerische Hochschulkonferenz (SHK) überführt.

\section{Situation seit 2015}

\section{Neue Rechtsgrundlagen}

Die unterschiedliche Trägerschaft der Eidgenössischen Technischen Hochschulen (ETH), kantonalen Universitäten, Fachhochschulen und Pädagogischen Hochschulen stellte für Kooperationsvorhaben zwischen Bibliotheken stets eine besondere Herausforderung dar. ${ }^{25}$ Seit 2015 beschreiten der Bund und die Kantone allerdings Neuland, indem sie, basierend auf dem Hochschulartikel in der Bundesverfassung (Art.63a BV), den tertiären Bildungsbereich gemeinsam för-

25 Die beiden Eidgenössischen Technischen Hochschulen werden vom Bund betrieben. Die zehn kantonalen Universitäten liegen im Verantwortungsbereich der jeweiligen Kantone. Die sieben öffentlich-rechtlichen Fachhochschulen werden jeweils von einem oder mehreren Kantonen getragen. Die Pädagogischen Hochschulen werden von den Kantonen finanziert. 
dern und koordinieren. Diese Neuordnung hat $\mathrm{zu}$ einer merklichen Vereinfachung im Aufbau und in der Durchführung gemeinsamer Fördervorhaben geführt. Folgende rechtliche Grundlagen sind für die Umsetzung dieses Verfassungsauftrages von Bedeutung (vgl. Abb. 5): ${ }^{26}$

- Bundesgesetz über die Förderung der Hochschulen und die Koordination im schweizerischen Hochschulbereich (Hochschulförderungs- und -koordinationsgesetz, HFKG): Das HFKG vom 30. September 2011 ist ein Koordinationsund Förderungsgesetz, das die Ziele und Grundsätze von Organisation und Verfahren der Koordination regelt. In Art. 59 HFKG werden Verwendungszweck und Voraussetzungen für projektgebundene Beiträge festgelegt: „,Mehrjährige projektgebundene Beiträge können für Aufgaben von gesamtschweizerischer hochschulpolitischer Bedeutung ausgerichtet werden. [...] Die an den Projekten beteiligten Kantone, Hochschulen und anderen Institutionen des Hochschulbereichs haben eine angemessene Eigenleistung zu erbringen.“ Die Inkraftsetzung des HFKG erfolgte in zwei Etappen: Die Bestimmungen betreffend Organe und Akkreditierung kamen am 01. Januar 2015 zur Anwendung, die neuen Finanzierungsbestimmungen gelten seit 01. Januar 2017. ${ }^{27}$

- Interkantonale Vereinbarung über den schweizerischen Hochschulbereich (Hochschulkonkordat): Für die Koordination im schweizerischen Hochschulbereich regelt das Hochschulkonkordat aus dem Jahr 2013 die Zusammenarbeit der Vereinbarungskantone untereinander und mit dem Bund. Die Inkraftsetzung erfolgte per 01. Januar 2015.

- Zusammenarbeitsvereinbarung zwischen Bund und Kantonen: Basierend auf dieser Vereinbarung wurden gemeinsame Organe geschaffen (siehe hierzu nächster Abschnitt). Jede Hochschule verfügt weiterhin über eine eigene Gesetzesgrundlage des jeweiligen Trägergemeinwesens.

26 Vgl. Die Interkantonale Vereinbarung über den schweizerischen Hochschulbereich (Hochschulkonkordat). Kurz-Info Generalsekretariat EDK, 26. Februar 2015 (http://www.edudoc.ch/ static/web/arbeiten/hsk_kurzinfo_d1.pdf).

27 Vgl. hierzu Informationen auf: Koordination Hochschulbereich, SBFI: https://www.sbfi.ad min.ch/sbfi/de/home/hs/hochschulen/koordination-hochschulbereich.html. 


\section{Bundesverfassung Art. 63a (Hochschulen)}

Bundesrecht:

Bundesgesetz HFKG

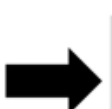

Zusammenarbeitsvereinbarung

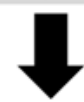

Schaffung gemeinsamer

Organe
Interkantonales Recht:

Hochschulkonkordat*
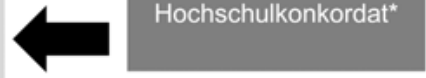

* Der Entscheid zum Beitritt obliegt jedem Kanton (kantonales Parlament).

Abb. 5: rechtlicher Rahmen der neuen Hochschulkoordination ${ }^{28}$

\section{Neue nationale Organstruktur}

Die Organstruktur wurde mit Inkraftsetzung des Hochschulförderungs- und -koordinationsgesetzes am 01. Januar 2015 gegenüber der früheren Situation wesentlich vereinfacht: Neu gibt es nur noch eine einzige (gemeinsame) Hochschulkonferenz, eine Rektorenkonferenz und einen Akkreditierungsrat (siehe Abb. 6). Diese Gremien ersetzen eine Vielzahl früherer Organe der universitären Hochschulen, Fachhochschulen und Pädagogischen Hochschulen.

28 Vgl. Interkantonale Vereinbarung (wie Anm. 26), hier nach Graphik 1. 


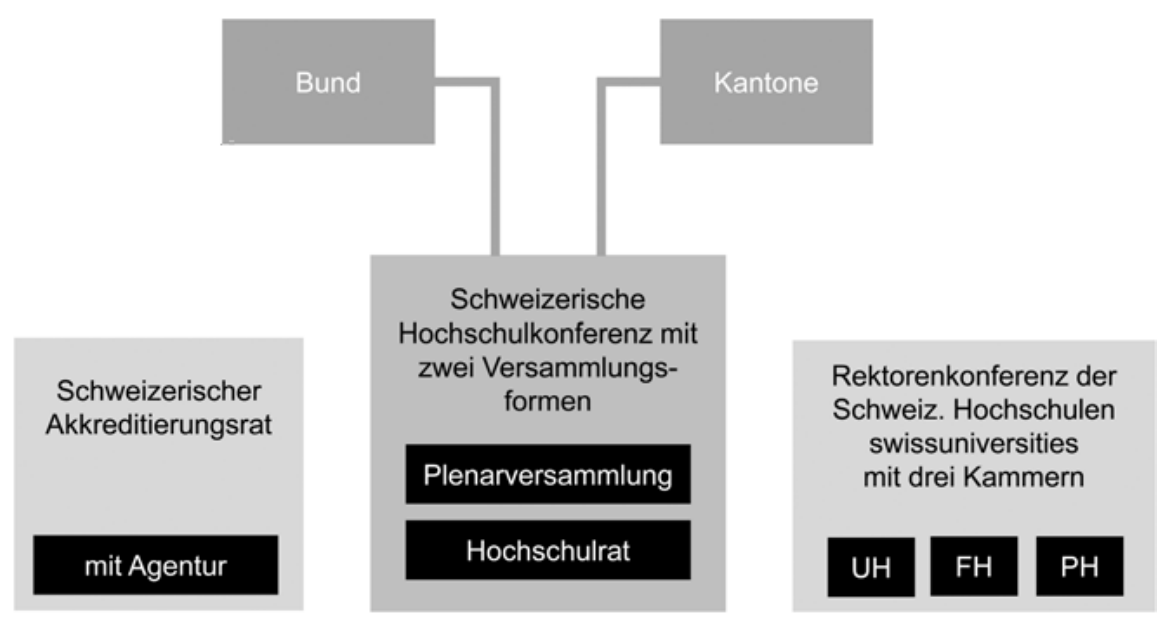

Abb. 6: neue Organstruktur seit $2015^{29}$

Schweizerische Hochschulkonferenz (SHK)

Die Schweizerische Hochschulkonferenz (SHK) ist seit 2015 das oberste hochschulpolitische Organ der Schweiz und sorgt für die gesamtschweizerische Koordination der Tätigkeiten von Bund und Kantonen im Hochschulbereich. Sie verfügt über rechtsetzende Kompetenzen, gibt Empfehlungen und Stellungnahmen $\mathrm{ab}$ und ist im Weiteren für die gesamtschweizerische hochschulpolitische Koordination und Aufgabenteilung in besonders kostenintensiven Bereichen zuständig. Die SHK tagt als Plenarversammlung oder als Hochschulrat. Der Bund bzw. der Bundesrat des Eidgenössischen Departements für Wirtschaft, Bildung und Forschung hat das Präsidium inne und ist zuständig für die Geschäftsführung.

Rektorenkonferenz swissuniversities

Die gemeinsame Rektorenkonferenz swissuniversities wurde 2015 als Verein gegründet und ist eine Zusammenführung der drei ehemaligen Rektorenkonferenzen CRUS (Universitäten), KFH (Fachhochschulen) und COHEP (Pädagogische Hochschulen). Swissuniversities setzt sich als gemeinsames Organ aller Hochschultypen für eine Vertiefung und Weiterentwicklung der Zusammenarbeit unter den schweizerischen Hochschulen ein und fördert eine gemeinsame Stim-

29 Vgl. Interkantonale Vereinbarung (wie Anm. 26), hier nach Graphik 2. 
me des Hochschulraums Schweiz. Die spezifischen Aufgaben der drei ursprünglichen Rektorenkonferenzen werden durch drei Kammern wahrgenommen.

Schweizerischer Akkreditierungsrat

Der Schweizerische Akkreditierungsrat besteht aus einem Expertengremium, das alle Schweizer Hochschulen nach einem einheitlichen Verfahren akkreditiert. Dieses Organ ist für die Bibliotheksarbeit kaum relevant.

\section{Nationale Organe für Bibliotheken}

\section{KUB: Kommission der Universitätsbibliotheken (1983-2000) bzw. Konferenz der Universitätsbibliotheken (seit 2001)}

Die Gründung der Kommission oder Konferenz der Universitätsbibliotheken (KUB) lässt sich auf das Jahr 1967 zurückverfolgen. ${ }^{30}$ Damals konstituierte sich die Gruppe der Hochschulbibliotheken innerhalb des Berufsverbands VSB neu als Direktorenkonferenz der Hochschulbibliotheken und widmete sich u.a. den Themen Koordination des Dissertationenaustausches, Zeitschriftenbeschaffung und Bibliotheksautomatisierung. 1983 wurde die Direktorenkonferenz durch die Kommission der Universitätsbibliotheken als Organ der damaligen Schweizerischen Hochschulkonferenz (SHK) ersetzt. Anlässlich der Reorganisation der SHK zur Schweizerischen Universitätskonferenz (SUK) im Jahre 2001 wurde sie bei gleichbleibendem Akronym zur Konferenz der Universitätsbibliotheken der Schweiz (KUB) umstrukturiert. ${ }^{31}$

Die KUB versteht sich als Koordinationsorgan der Schweizer Hochschulbibliotheken, das sich mit allen Aspekten des wissenschaftlichen Bibliothekswesens befasst. Hierbei stehen strategische Überlegungen im Vordergrund; aber auch Detailfragen, die hohes Spezialwissen erfordern, werden hier behandelt, so z. B. die Langzeitarchivierung oder das Urheberrecht. Die KUB hat im Wesentlichen die Aufgabe eines Diskussionsforums. Sie kann in der heutigen Form zwar keine für alle verbindlichen Entscheidungen treffen, hat aber normativen Charakter für bibliothekarische Fragestellungen. Zudem fungiert sie als Auftraggeberin für einzelne nationale Projekte, z.B. das Konsortium.

30 Vgl. Barth/Schneider, Bibliotheken (wie Anm. 2), S. 48.

31 Vgl. Noëmi Eglin-Chappuis: Governance von Universitätsbibliotheken: Optimierungsmöglichkeiten aus institutioneller Sicht. Chavannes-Lausanne 2009 (Cahier de l'IDHEAP 245), S. 18. 
Die Website der KUB informiert über laufende und abgeschlossene Projekte. ${ }^{32}$ $\mathrm{Zu}$ den laufenden Projekten gehören die Unterstützung der nationalen Open Access-Strategie ${ }^{33}$, der Aufbau der Swiss Library Service Platform (SLSP), die Weiterentwicklung des Konsortiums sowie die Kooperative Print-Archivierung (KPA) - ein Kooperationsprojekt zur sicheren, langfristigen Aufbewahrung eines gedruckten Exemplars von wissenschaftlichen ausländischen Zeitschriften.

Unter den abgeschlossenen Projekten sind u.a. aufgelistet: elektronische Dissertationen (Kooperationsprojekt mit der Nationalbibliothek zur digitalen Langzeitarchivierung), die Einführung von RDA (internationales Regelwerk für die Katalogisierung) oder BibliOpass (gegenseitige Anerkennung von Benutzungsausweisen). Ausserdem hat die KUB verschiedene Stellungnahmen verfasst ${ }^{34}$, so beispielsweise zur Revision des Urheberrechtsgesetzes (2016) oder zum Entscheid des Bundesgerichtes zum Dokumentenlieferdienst der Bibliotheken (2014). Die KUB verfügt auch über mehrere Arbeitsgruppen. $\mathrm{Zu}$ erwähnen sind der Arbeitskreis Open Access (AKOA) als offizielles Expertengremium oder die Arbeitsgruppe zur Kooperativen Print-Archivierung.

Nebst den genannten Projekten und Themen betrifft eine der zentralen Fragen die künftige stärkere Einbettung der KUB in die Schweizer Hochschullandschaft. Die aktuelle Unabhängigkeit der KUB ist zum einen eine ihrer Stärken, denn sie lässt die KUB relativ flexibel handeln, zum anderen stösst die KUB damit auch an ihre Grenzen, bleibt ihr Einfluss über die Hochschulbibliotheken hinaus doch relativ beschränkt. Angesichts der zunehmenden Vernetzung der Schweizer Hochschullandschaft, der Schaffung diverser nationaler Kooperationsstrukturen und der zunehmenden Realisierung von Kooperationsprojekten hat die KUB ein Interesse daran, als Expertengremium stärker in die nationalen Hochschulstrukturen (swissuniversities) eingebunden zu werden. Aus Sicht von Niklaus Landolt, Direktor der Universitätsbibliothek Bern, kann die KUB ihrer wichtigen Rolle innerhalb der Schweizer Hochschullandschaft nur dann gerecht werden, wenn sie fester und integrierter Bestandteil dieses nationalen Netzwerks wird. ${ }^{35}$

32 Vgl. http://www.kub-cbu.ch/projekte-projets/.

33 Vgl. hierzu den Beitrag „Nationale Open Access-Strategie“ von Christian Fuhrer und René Schurte in diesem Band.

34 Vgl. http://www.kub-cbu.ch/dokumente-documents/. Hier findet man auch weiterführende Informationen zu den Arbeitsgruppen AKOA und KPA.

35 Die Autorin dankt Niklaus Landolt für seinen Input zu diesem Abschnitt. 


\section{Swiss Library Service Platform (SLSP)}

Der Aufbau einer gemeinsamen Serviceplattform gilt aktuell zweifellos als grösstes und ambitioniertestes Bibliotheksprojekt der Schweiz. Unter dem Namen „Swiss Library Service Platform“ soll einerseits eine Governancestruktur für Hochschulbibliotheken der Schweiz und andererseits eine leistungsfähige Serviceplattform, basierend auf einer gemeinsamen Bibliothekssoftware, aufgebaut werden. Die gleichnamige Aktiengesellschaft wurde am 30. Mai 2017 gegründet. Gründungsaktionäre sind 15 Institutionen aus dem Hochschulbereich der Schweiz. Das Projekt wird im Beitrag von Wolfram Neubauer in diesem Band ausführlich vorgestellt.

\section{BIS}

Der BIS ist der nationale Verband für Bibliotheken, Informations- und Dokumentationsstellen und deren Mitarbeitende. ${ }^{36}$ Für Bibliotheken unterschiedlicher Art versteht er sich als politische Stimme und stärkt ihre Rolle in der Gesellschaft. Darüber hinaus sichert er die berufliche Grund- und Weiterbildung, sorgt für die Vernetzung seiner Mitglieder und fördert deren kontinuierliche Professionalisierung und Weiterentwicklung. Der BIS veröffentlicht die schweizerische Statistik der Bibliotheken und die Zeitschrift arbido. Die Aktivitäten des BIS werden im Beitrag von Herbert Staub im vorliegenden Band vorgestellt.

\section{Zusammenfassung}

Der vorliegende Beitrag zeigt den Umfang und die Komplexität der für die Wissenschaftlichen Bibliotheken der Schweiz relevanten nationalen Förderstrukturen. Zur Vielschichtigkeit tragen einerseits die verschiedenen Hochschultypen (universitäre Hochschulen, Fachhochschulen, Pädagogische Hochschulen) und die unterschiedlichen Trägerschaften (Bund oder Kanton) bei. Andererseits ist die Schweiz auch ein mehrsprachiges Land, was eine weitere Herausforderung für die Zusammenarbeit darstellt. Mit der Inkraftsetzung der neuen Rechtsgrundlagen und der Neustrukturierung der nationalen Organe verfügen die Hochschulen und damit auch die Bibliotheken seit 2015 über ein gemeinsames Dach, was $\mathrm{zu}$ einer signifikanten Vereinfachung bei der Initiierung und Umsetzung von

36 Vgl. http://www.bis.ch/verband/ueber-uns.html. 
Projekten beiträgt. Darüber hinaus steht den Bibliotheken seit der Gründung der Aktiengesellschaft SLSP eine eigene Rechtspersönlichkeit für den Aufbau und Betrieb einer gemeinsamen Serviceplattform zur Verfügung.

Die kooperative Planung und Durchführung von Projektvorhaben setzt allerdings nebst diesen rechtlichen und strukturellen Rahmenbedingungen auch eine solide finanzielle Basis voraus. Hier profitieren die Bibliotheken seit dem Jahr 2000 ganz erheblich von nationalen projektgebundenen Beiträgen. Das Ausmass und die inhaltliche Vielfalt dieser Kooperationen sind beachtlich und zeigen auch das Entwicklungspotenzial der Wissenschaftlichen Bibliotheken der Schweiz.

\section{Anhang}

Tabelle 2 bietet eine Übersicht über die Vielzahl der Bibliotheksprojekte, die im Rahmen nationaler Förderprogramme zwischen 2000 und 2017 erfolgreich beantragt und durchgeführt worden sind: ${ }^{37}$

\begin{tabular}{ll}
\hline \multicolumn{2}{l}{ Förderprogramm: Konsortialprojekte (2000-2007) } \\
\hline Konsortium: Lizenzen & Lizenzierung von Zeitschrifteninhalten \\
\hline Konsortium: E-Archiving & Digitalisierung, Langzeitarchivierung, Metadatenserver \\
\hline Förderprogramm: e-lib.ch (2008-2012) \\
\hline Konsortium: E-Depot & Service zur zentralen Speicherung lizenzierter Inhalte \\
\hline Info-Net Economy & Dokumentations- und Informationsnetzwerk für die \\
& Wirtschaftswissenschaften \\
\hline ACCEPT & Entwicklung nutzungsrelevanter Kriterien für digitale \\
& Bibliotheken und Ermittlung der Benutzerakzeptanz für die \\
& Produkte von e-lib.ch \\
\hline ElibEval & Gesamtkonzept für die Usability-Evaluation von Online- \\
& Angeboten innerhalb von e-lib.ch: Elektronische Bibliothek \\
\hline swissbib & Schweiz \\
\hline
\end{tabular}

37 Entsprechend der Website von swissuniversities wird für die Periode ab 2013 zwischen abgeschlossenen und laufenden Projekten unterschieden. Es handelt sich hierbei um Projekte im Rahmen der Förderprogramme SUK P-2 und P-5 (Stand Juni 2017). Quellen: http://www.e-lib.ch/ copy/de/content/download/1712/17250/version/8/file/laufblatt_ger.pdf und https://www.swiss universities.ch/de/organisation/projekte-und-programme/p-5/abgeschlossene-projekte/. 


\begin{tabular}{|c|c|}
\hline Webportal e-lib.ch & $\begin{array}{l}\text { zentrales Webportal für eine schweizweite Informations- } \\
\text { bereitstellung }\end{array}$ \\
\hline Marketing e-lib.ch & $\begin{array}{l}\text { Marketingkonzept und -massnahmen für die Angebote von } \\
\text { e-lib.ch }\end{array}$ \\
\hline Kartenportal.CH & $\begin{array}{l}\text { Aufbau einer virtuellen Fachbibliothek „Geodaten und Karten“ } \\
\text { mit Metainformationen zu Geodatenangeboten und einer } \\
\text { zentralen Kartensuche }\end{array}$ \\
\hline $\begin{array}{l}\text { Konsolidierung des } \\
\text { Metadatenservers }\end{array}$ & $\begin{array}{l}\text { Integration des Metadatenservers aus dem Projekt } \\
\text { E-Archiving in die Suchmöglichkeiten von e-lib.ch sowie } \\
\text { Einbindung von Metadatenbeständen }\end{array}$ \\
\hline RODIN & $\begin{array}{l}\text { Roue d'Information: Werkzeug zur Bündelung und Koppelung } \\
\text { benutzerrelevanter Informationsquellen }\end{array}$ \\
\hline e-codices & Virtuelle Handschriftenbibliothek der Schweiz \\
\hline Best Practices Digitalisierung & Publikation von „Best Practices“ für Digitalisierungen \\
\hline $\begin{array}{l}\text { Öffnung der Plattform retro. } \\
\text { seals.ch für regionale Inhalte }\end{array}$ & $\begin{array}{l}\text { Retrodigitalisierung wissenschaftlicher Zeitschriften mit } \\
\text { regionalem Charakter }\end{array}$ \\
\hline e-rara.ch & $\begin{array}{l}\text { Online-Plattform für digitalisierte alte Drucke aus Schweizer } \\
\text { Bibliotheken }\end{array}$ \\
\hline $\begin{array}{l}\text { Multivio - generic browser and } \\
\text { visualizer for digital objects }\end{array}$ & Universalwerkzeug für den Zugriff auf digitale Inhalte \\
\hline $\begin{array}{l}\text { Informationskompetenz an } \\
\text { Schweizer Hochschulen }\end{array}$ & $\begin{array}{l}\text { Standards zur Informationsvermittlung und Schaffung eines } \\
\text { virtuellen Kompetenzzentrums }\end{array}$ \\
\hline $\begin{array}{l}\text { Recherchekompetenz im } \\
\text { Bereich wissenschaftlicher } \\
\text { Suchmaschinen }\end{array}$ & $\begin{array}{l}\text { Auf- und Ausbau der Recherchekompetenz von Kundinnen } \\
\text { und Kunden der Elektronischen Bibliothek Schweiz bei der } \\
\text { Suche nach Informationen über spezielle Suchmaschinen }\end{array}$ \\
\hline Konzept Langzeitarchivierung & $\begin{array}{l}\text { Entwicklung eines Modells für eine zentrale } \\
\text { Langzeitarchivierung von digitalen Primär- und } \\
\text { Sekundärdaten für die Schweiz }\end{array}$ \\
\hline $\mathrm{DOI}-\mathrm{CH}$ & $\begin{array}{l}\text { Aufbau einer zentralen DOI-Vergabestelle für alle Schweizer } \\
\text { Hochschulen }\end{array}$ \\
\hline \multicolumn{2}{|c|}{ Förderprogramm: SUK P-2 (abgeschlossene Projekte) } \\
\hline SYMPHONY & $\begin{array}{l}\text { Swiss System for Monitoring Bibliographic Data and Holistic } \\
\text { Publication Behavior Analysis }\end{array}$ \\
\hline HOPE for Open Access & $\begin{array}{l}\text { Hauptbibliothek Open Publishing Environment: Publikation } \\
\text { wissenschaftlicher Zeitschriften mit Open Access }\end{array}$ \\
\hline e-codices & Virtuelle Handschriftenbibliothek der Schweiz \\
\hline AISOOP & Studie: Schichtenmodell zu Online-Plattformen \\
\hline
\end{tabular}




\begin{tabular}{|c|c|}
\hline Pilot-ORD @ CH & Schweizer Publikationsplattform für offene Forschungsdaten \\
\hline $\begin{array}{l}\text { Research Data Life-Cycle } \\
\text { Management (Vorprojekt) }\end{array}$ & Grundlagenprojekt Forschungsdaten \\
\hline Swiss edu-ID (beta) & Next-generation Swiss Educational Identity Management \\
\hline SLSP & Swiss Library Service Platform (Konzeptphase) \\
\hline SCALE & Swiss Cloud for Academic and Learning Experts \\
\hline DICE & $\begin{array}{l}\text { Network of Competence \& Training in Legal Issues for Digital } \\
\text { Contents }\end{array}$ \\
\hline Geodata4SwissEDU & $\begin{array}{l}\text { Aufbau, Betrieb und Weiterentwicklung des nationalen } \\
\text { Service geodata4edu.ch }\end{array}$ \\
\hline $\begin{array}{l}\text { Kooperative Speicherbibliothek } \\
\text { Schweiz }\end{array}$ & $\begin{array}{l}\text { Gemeinschaftsprojekt für eine kooperative } \\
\text { Speicherbibliothek }\end{array}$ \\
\hline \multicolumn{2}{|c|}{ Förderprogramm: SUK P-2 und P-5 (laufende Projekte) } \\
\hline Nationallizenzen & $\begin{array}{l}\text { Lizenzierung lückenloser Archive elektronischer Ressourcen } \\
\text { an den Schweizer Hochschulen }\end{array}$ \\
\hline jemr.org & $\begin{array}{l}\text { Anpassung und Relaunching eines transdisziplinären } \\
\text { Fachjournals auf die Plattform BOP (Bern Open Publications) }\end{array}$ \\
\hline e-codices & $\begin{array}{l}\text { Virtuelle Handschriftenbibliothek der Schweiz } \\
\text { (Produktionsphase) }\end{array}$ \\
\hline e-rara.ch: Volltext & Erweiterung von e-rara.ch um Texterkennung (OCR) \\
\hline e-manuscripta.ch: Volltext & Nachnutzung für die Wissenschaft - der Weg zum Volltext \\
\hline NIE-INE & $\begin{array}{l}\text { Nationale Infrastruktur für Editionen - Infrastructure } \\
\text { nationale pour les éditions }\end{array}$ \\
\hline histHub & $\begin{array}{l}\text { Datenhub und Laboratory für Linked Open Data zu } \\
\text { historischen Entitäten der Schweiz }\end{array}$ \\
\hline swissbib & Schweizer Metakatalog (Betrieb und Ausbau) \\
\hline $\begin{array}{l}\text { linked.swissbib.ch (Beta) / } \\
\text { REST API (Beta) }\end{array}$ & Integration von swissbib in das semantische Web \\
\hline $\begin{array}{l}\text { Research Data Life-Cycle } \\
\text { Management }\end{array}$ & From Pilot Implementations to National Services \\
\hline MedRed@HES-SO & Medical Research Data Acquisition Platform \\
\hline Data Analysis Service & $\begin{array}{l}\text { Data analysis infrastructure for the multi-disciplinary users } \\
\text { of PSI large scale facilities }\end{array}$ \\
\hline eScience Coordination Team & Formation of a national coordination service \\
\hline EnhanceR & Enhancing Research through IT Expertise \\
\hline
\end{tabular}




\begin{tabular}{ll}
\hline Train2Dacar & $\begin{array}{l}\text { Entwicklung der Ausbildung im Bereich des } \\
\text { Forschungsdatenmanagements }\end{array}$ \\
\hline Swiss edu-ID P-II & Swiss edu-ID Phase II \\
\hline Swiss edu-ID Depl1 & Swiss edu-ID Deployment Step 1 \\
\hline Swiss edu-ID Mobile & Swiss edu-ID Mobile App \\
\hline SLSP & Swiss Library Service Platform (Aufbauphase) \\
\hline SCALE-UP & Services for the Swiss Cloud for Academic and \\
\hline Learning Experts
\end{tabular}

